\title{
Results from a spectroscopic survey in the CoRoT fields
}

\section{Search for chromospherically active stars ${ }^{\star} \star \star$}

\author{
B. J. Medhi ${ }^{1,2}$, S. Messina ${ }^{3}$, Padmakar S. Parihar ${ }^{4}$, I. Pagano ${ }^{3}$, S. Muneer ${ }^{5}$, and K. Duorah ${ }^{1}$ \\ 1 Department of Physics, Gauhati University, Assam 781014, India \\ e-mail: bimanjm@yahoo.com \\ 2 Aryabhatta Research Institute of Observational Sciences (ARIES), Manora Peak, Nainital 263129, India \\ 3 INAF - Catania Astrophysical Observatory, via S. Sofia 78, 95123 Catania, Italy \\ e-mail: [sme;ipa]@oact.inaf.it \\ ${ }^{4}$ Indian Institute of Astrophysics, CREST, Block II, Koramangala, Bangalore 560034, India \\ e-mail: psp@iiap.res.in \\ 5 Indian Institute of Astrophysics, VBO, Bangalore, India \\ e-mail: muneer_melathil@yahoo.com
}

Received 8 September 2005 / Accepted 12 April 2007

\section{ABSTRACT}

\begin{abstract}
Aims. In this paper we report the results of the spectroscopic survey we carried out to detect chromospheric activity of late-type stars in the fields of view of the CoRoT space mission.

Methods. We give an accurate MK classification of all targets, which is valuable information on both the main CoRoT project and additional science programs, by means of cross-correlation with MK standard stars, by using artificial neural networks. The presence or absence of excess $\mathrm{H} \alpha$ emission, determined using spectral subtraction technique, is used to characterize the chromospheric activity level.

Results. In most cases our MK classification agrees with the spectral classification reported in the SIMBAD database; however, there are a few stars that are found to have very different MK classes. Our survey reveals that $\sim 7 \%$ of late-type stars in our sample indeed possess a very active chromosphere. The measured $\mathrm{H} \alpha$ excess and the presence of the $6708 \AA \mathrm{Li}$ line allow us to confirm the membership of 5 targets in the young open cluster NGC 2264.
\end{abstract}

Key words. stars: late-type - stars: activity - stars: fundamental parameters

\section{Introduction}

CoRoT (Convection Rotation and Planetary Transits) is a small optical space telescope dedicated to asteroseismology and to the search for extrasolar planets (Baglin et al. 2000) and put in orbit on Dec. 27, 2006. In the coming years CoRoT will provide ultra-high precision photometry for thousands of stars, with a temporal resolution from $8 \mathrm{~min}$ down to $1 \mathrm{~s}$ and up to 150 days long. The CoRoT observable skies are two regions of $10 \mathrm{deg}$ radius located at the intersection of the galactic plane with the celestial equator: RA $=18^{\mathrm{h}} 50^{\mathrm{m}}, \mathrm{Dec}=0^{\circ}$ (in the direction of the galactic center) and RA $=06^{\mathrm{h}} 50^{\mathrm{m}}$, Dec $=0^{\circ}$ (anti-center direction). The central program of asteroseismology aims at observing at least 6 different sets of target stars very precisely over a period of 150 days each, whereas under the exoplanet program, CoRoT will provide extremely long and uninterrupted sequences of photometric data on more than one hundred thousand stars of $V$ magnitude between 12 and 16. In addition to asteroseismology and the search for extrasolar planets, the data provided by CoRoT are expected to be extremely useful for

* Based on observations collected at the Himalayan Chandra Telescope of the Indian Institute of Astrophysics, India.

$\star \star$ Tables 1-5 and Figs. 7-12 are only available in electronic form at the CDS via anonymous ftp to

cdsarc.u-strasbg.fr $(130.79 .128 .5)$ or via

http://cdsweb.u-strasbg.fr/cgi-bin/qcat?J/A+A/469/713 investigating chromospherically active stars, QSOs, X-ray binaries, white dwarfs, cataclysmic variables, pre-main sequence Ae-Be stars, and magnetic Ap stars (Gilmore 2001; Weiss et al. 2003). For instance, stellar magnetic activity, which is a potential source of noise in the detection of planetary transits (see e.g., Lanza et al. 2003, 2005; Moutou et al. 2005), will be studied in the context of the CoRoT additional programs. In fact, the ultraprecise CoRoT photometric data can be used to study the convection, properties, and evolution of photospheric active regions (Aigrain et al. 2004; Lanza et al. 2003, 2004), stochastic variability such as micro-flaring, stellar rotation, and surface differential rotation of late-type stars.

To gather as much a priori information as possible on the potential target stars, i.e., fundamental stellar physical parameters and peculiarities such as photometric or spectroscopic variability, and to maximize the scientific outcome of CoRoT data, an ambitious ground-based observing program, of obtaining Strömgren photometry and high-resolution spectroscopy for more than 1500 objects was carried out by several teams within the framework of the CoRoT working groups for ground-based observations (Solano et al. 2005; Poretti et al. 2003, 2005). The characterization of magnetic activity of CoRoT asteroseismology primary targets was carried out at Catania Observatory by monitoring the Ca II H\&K lines (Pagano et al., in preparation). Here, we report the results of our spectroscopic observations of a sample of late-type stars in order to identify new 

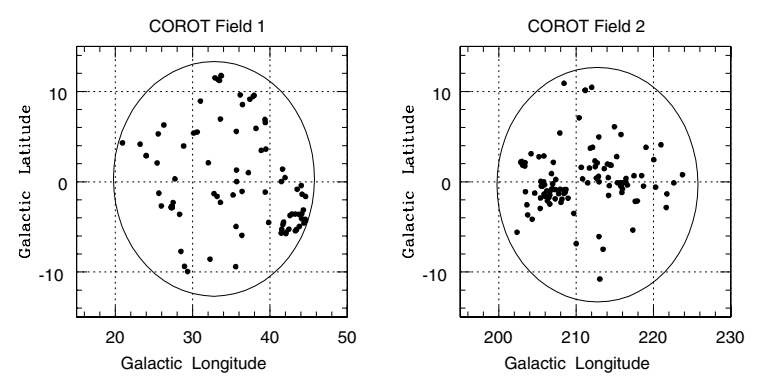

Fig. 1. The observed bright and weak stars in the galactic center and anti-center fields of view of the CoRoT space telescope.

chromospherically active stars to be proposed as potentially rewarding targets for the CoRoT Additional Programs. The paper is organized as follows: in Sect. 2 we discuss our target selection criteria and give brief information about the instruments, observation, and reduction technique. In Sect. 3, we discuss the analysis methods and give results on the spectral classification and $\mathrm{H} \alpha$ behavior. The conclusions are given in Sect. 4.

\section{The data}

Our main stellar sample, hereafter referred to as the bright stars sample (BS), was selected according to the following criteria: a) stars in the CoRoT fields of view, i.e. in circles having $10^{\circ}$ radius and centered on RA $=18^{\mathrm{h}} 50^{\mathrm{m}}$, Dec $=0^{\circ}$ (galactic center direction) and RA $=06^{\mathrm{h}} 50^{\mathrm{m}}$, Dec $=0^{\circ}$ (galactic anticenter direction), respectively; $b$ ) $6.0 \leq V \leq 9.5 \mathrm{mag}$, according to the observational constraints of the CoRoT asteroseismology program; and $c)$ late-type stars $(0.58 \leq B-V \leq 2.00)$ for which some degree of magnetic activity can be expected. As determined from the Tycho 2.0 catalog (Hog et al. 2000), which is complete at $99 \%$ level for stars brighter than $V=11.0$, the BS sample turned out to have $\sim 2260$ targets. We report here on a sub-sample of 150 bright stars ( $\sim 6.6 \%$ of the sample) randomly selected among the complete BS sample: the target identification, coordinate, $V$ magnitude, and SIMBAD spectral type are given in Table 1, together with each target observation date. A second sample of targets, hereafter referred to as the weak stars sample (WS), has been selected to test the capability of our instrumental setup and analysis tools to detect the presence of chromospheric activity and to determine accurate MK spectral classes also on targets of interest to the CoRoT exoplanetary program. This sample is made of 30 targets selected according to the above $a$ ) and $c$ ) criteria but with $11.0 \leq V \leq 16.0$ mag (see Table 2 for information on WS targets). Among many candidate targets that satisfied these selection criteria, we preferentially selected those which were already known to be chromospherically active. The location of the observed BS and WS target stars is shown in Fig. 1 in the galactic coordinate system. Our sample is complete neither for the BS sample nor (and here is strongly incomplete) for stars in the range $V=12-16$.

Our spectroscopic survey was carried out on a total of 11 nights during the years 2003-2004 using the $2 \mathrm{~m}$ HCT (Himalayan Chandra Telescope) of the Indian Astronomical Observatory, equipped with the HFOSC spectrograph $^{1}$. We made use of the $\mathrm{H} \alpha$ line as chromospheric activity diagnostic, rather than the $\mathrm{Ca}$ II $\mathrm{H} \& \mathrm{~K}$ lines, the instrumental response being very poor in the blue spectral region. We used the red grism Gr8, which covers nearly a $4000 \AA$ spectral

1 More details on the HFOSC instrument may be obtained at http://www.iiap.res.in/iao_hfosc.htm window starting from $5400 \AA$ and which gives a dispersion of about $1.3 \AA$ /pixel. The spectrograph slit width was fixed at 67 micron $\left(\sim 0.75^{\prime \prime}\right)$. The instrumental spectral resolution, as derived from the 2.5 pixels FWHM of sharp Fe and Ne lines, resulted in $\Delta \lambda \sim 3.25 \AA(R \sim 2000)$. The optimum exposure time, ranging from a few tens to a few hundreds seconds, was chosen to typically achieve a 100-300 signal-to-noise ratio. During our observations, the seeing value varied between 1.2 and 2.5 arcsec. To estimate and to correct for atmospheric extinction effects, we observed several spectroscopic standard stars from the STELIB catalog (Le Borgne et al. 2003) each night, and then compared them with program star spectra. We also observed a large number of chromospherically inactive stars from Montes et al. (1995, 1997) and Montes et al. (1999) using the same instrumental setup as used for the program stars. On each night, observations of at least one spectrophotometric standard close to the CoRoT fields were also carried out with the purpose of flux calibration. The spectroscopic observations were reduced and analyzed with the CCD image reduction package $c c d r e d$ and spectroscopic reduction package specred available within IRAF. After bias subtraction and flat field correction, stellar spectra were optimally extracted. A FeNe arc lamp was used for the wavelength calibration, and, finally, the flux calibration was done using spectrophotometric standard stars.

\section{Results}

In Sect. 3.1, we give the description and the results of the two classification methods we adopted to infer the MK spectral class of our program stars. In Sect. 3.2, we describe the spectral subtraction technique we adopted to identify which stars in our sample are chromospherically active. Then, we extend to the coronal level our investigation on magnetic activity by computing the X-ray luminosities from archival data. In Sect. 3.3, we focus our investigation on 5 stars in our sample which are members of the young open cluster NGC 2264.

\subsection{The spectral classification}

We used two different techniques to determine an accurate MK spectral type for the program stars, i.e. the cross-correlation between the program and MK standard star spectra, and the artificial neural network (ANN). A brief description of these two techniques and the results we obtained are given below.

\subsubsection{Classification using cross-correlation technique}

This is a classical method used in spectral MK classification work. In this technique, the cross-correlation of an unknown program's stellar spectrum is done with a sample of MK standard stellar spectra covering a range of spectral types and luminosity classes. Since the instrumental profile in the spectrum of the program star may degrade the classification accuracy, we used spectra of program and standard stars obtained from the same instrumental setup. To have a complete sample of MK standards as possible, we decided to use two other sets of standard star spectra taken from the STELIB library of stellar spectra and the Atlas of Southern MK Standards (5800-10200 ̊) by Danks \& Dennefeld (1994). Our final grid of standard stars includes almost all subtypes from F0V to M3V, from F6IV to K1IV, from F0III to M7III, from G5II to K3II, and from F0I to M2I. Imperfect flux calibration, interstellar reddening, and atmospheric dispersion can all distort the shape of the stellar 
continuum. Therefore, we decided to normalize the spectrum with respect to the continuum using the median and boxcar filtering procedure as described by Bailer-Jones (1997) and Bailer-Jones et al. (1998). In the following step, the spectral resolution of the program and standard spectra were matched by convolving one or both spectra with a Gaussian kernel. To remove from our analysis those regions severely affected by telluric lines (6850-7360, 7560-7730, 8100-8400 ̊), as well for consistency with the spectral range used with the ANN method described in the next section, our classification was achieved by considering only the 5450-6800 $\AA$ spectral region. Both target and standard spectra were re-binned using quadratic spline interpolation, and the normalized flux was computed in the same wavelength bin. Finally, individual reference spectra from the catalogs of standards were cross-correlated with program stars. In order to also take the rotational velocity of the targets into account, each standard spectrum was rotationally broadened within a range of $v \sin i$ values from 0 to +50 with a step of $3 \mathrm{~km} \mathrm{~s}^{-1}$ (Frasca et al. 2003). The quality of the match was judged by considering the correlation coefficients between program and standard spectra with different rotational velocity broadenings. The standard spectrum giving the highest correlation coefficient was used to assign the MK class. In Cols. 2-3 of Table 3 we list the final MK class and correlation coefficient as derived from the cross-correlation technique. A comparison with the spectral classes provided either by SIMBAD and assigned by ANN for a sample of standard stars shows that the cross-correlation technique is capable of assigning the correct luminosity class to dwarfs and subgiants and with an accuracy of one class to giants and supergiants and of assigning the spectral type with an accuracy of about 2 sub-types.

\subsubsection{Classification using artificial neural network}

To obtain accurate MK class of CoRoT stars we also used a supervised artificial neural network (ANN) code based on a multilayer back propagation algorithm. For a detailed description of the ANN code and its use, we refer the reader to, e.g., Gulati et al. (1994) and Singh et al. (1998). Unlike the ANN used by Bailer-Jones et al. (1998), where the output node can be further subdivided (spectral type and luminosity class), the ANN code developed by Gulati et al. (1994) has only one output node. The output coding scheme used in this ANN program gives maximum weight to spectral type, while the luminosity class gets only one tenth the weight of spectral subclasses, which is why we had difficulty in classifying luminosity class reliably. To avoid this problem we followed the suggestion of Bailer-Jones et al. (1998) and split the networks into two separate networks, one for spectral type and the other for luminosity class. A total of 35 spectra of G-M type and of III-V luminosity class were taken into consideration while training the network. The trained networks were tested with 20 known spectra and the RMS of the difference between ANN classification and the known one was used to judge the quality of the classification. The red spectra are heavily affected by telluric lines whose strength varied during observing periods. In the beginning, we tried to use whole red spectrum in the range of 5400 to $9100 \AA$ and then masked data at the regions severely affected by telluric lines (6850-7360, 7560-7730, 8100-8400 ^). Though there was improvement in the MK classification accuracy after masking the data, the best MK classification was achieved by considering only the 5450-6800 ^ spectral region. Furthermore, from earlier works (Singh et al. 1998; Bailer-Jones et al. 1998), it was found that

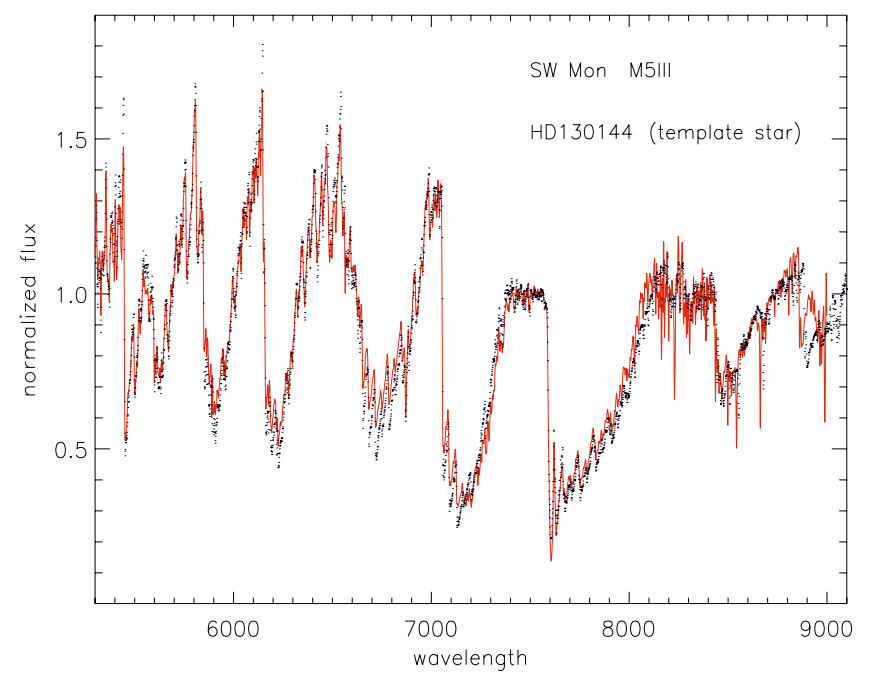

Fig. 2. The observed spectrum of SW Mon (dots) with the template spectrum of the M5 III star HD 130144 (solid line) overplotted.

reduction in the dimensionality of input data helps to enhance the reliability of the neural network. Therefore, we also tried to use principal component analysis (PCA) and reduced the dimensionality with various factors; but we did not find much improvement in the classification accuracy, so we decided to use ANN without PCA. The lowest RMS error of 1.85 sub-classes was achieved in the spectral type classification. However, in the case of discrete luminosity classes, the network outputs are probabilities; and following Bailer-Jones et al. (1998) we constructed a confusion matrix. The elements of the confusion matrix give an idea of what percentage of spectra have been correctly and incorrectly classified. From Table 4 it appears that the network could correctly classify luminosity class III and V, but it failed to do the same with class VI. In our ANN-based MK classification, we did not try to determine stellar metallicity, and we chose our standard stars with a metallicity close to the solar value $(Z=0.019)$. In Cols. 4, 5 of Table 3, we list the final MK class and correlation coefficient as derived from the ANN method.

\subsubsection{Final MK class assignment}

As shown in Table 3, cross-correlation and ANN methods assign similar MK spectral types (within a difference of 2-3 subclasses) to more than $90 \%$ of our targets and the same luminosity class to almost $65 \%$ of our targets. In Fig. 2 we show as an example the case of SW Mon to which both methods assign the same spectral class. The cross-correlation method tends to give higher correlation coefficient values with respect to ANN. In order to confirm the MK spectral class or to assign the correct one in those cases for which cross-correlation and ANN methods gave different results, we compared the target's absolute magnitude and $B-V$ color with a grid of theoretical evolutionary tracks (see Fig. 3) for a range of masses and metallicities $(Z=0.0004-0.03)$, as taken from Girardi et al. (2000). Specifically, we used $J H K$ magnitudes from the 2MASS All Sky Survey (Cutri et al. 2003) and $J-H$ vs. $H-K$ color diagrams in the Johnson-Glass system, as established by Bessel \& Brett (1988), to determine the color excess, and the Hipparcos parallax (Perryman et al. 1998) to compute the target's absolute magnitude. For 73 out of 180 targets, the parallax and the $J H K$ magnitudes existed and were accurate enough to constrain the spectral class. Absolute magnitudes and colors resulted in 
Table 6. The 20 stars showing $\mathrm{H} \alpha$ excess.

\begin{tabular}{|c|c|c|c|c|c|c|c|c|}
\hline $\begin{array}{c}\text { Active } \\
\text { program star }\end{array}$ & $\bar{V}$ & Sp.type & $\begin{array}{l}\text { Inactive } \\
\text { comp. star }\end{array}$ & Sp.type. & $\begin{array}{c}\mathrm{H} \alpha E W \\
(\AA)\end{array}$ & X-ray activity & Note & $\begin{array}{c}\text { New/known } \\
\text { chromospheric emission }\end{array}$ \\
\hline HD 170493 & 8.05 & K3V & HD 219134 & K3V & 0.1 & & & new \\
\hline HD 175905 & 7.66 & K0III & HD 188947 & K0III & 0.2 & & & new \\
\hline HD 168159 & 9.29 & $\mathrm{~K} 3 \mathrm{~V}$ & HD 219134 & $\mathrm{~K} 3 \mathrm{~V}$ & 0.3 & $\log \frac{L_{X}}{L_{\ln l}}=-3.91$ & $\mathrm{a}$ & known \\
\hline HD 175545 & 7.40 & K0III & HD 188947 & K0III & 0.3 & & & new \\
\hline NGC 2264 VAS 10 & 11.67 & K0IB & HD 206778 & $\mathrm{~K} 2 \mathrm{Ib}$ & 0.3 & & & new \\
\hline NGC 6705602 & 11.46 & K0.5II & HD 164349 & K0.5II & 0.3 & & & new \\
\hline HD 178126 & 9.22 & $\mathrm{~K} 3 \mathrm{~V}$ & HD 219134 & K3V & 0.4 & & & new \\
\hline HD 262323 & 8.75 & K0Ibvar & HD 206778 & $\mathrm{~K} 2 \mathrm{Ib}$ & 0.4 & & & new \\
\hline HD 52919 & 8.34 & $\mathrm{~K} 2 \mathrm{~V}$ & HD 4628 & $\mathrm{~K} 2 \mathrm{~V}$ & 0.5 & $\log \frac{L_{X}}{L_{\mathrm{b} \mid}}=-3.78$ & a & new \\
\hline HD 46122 & 7.32 & G6IV & HD 190360 & G6IV & 0.7 & $\log \frac{L_{X}^{L}}{L_{\mathrm{bol}}}=-3.21$ & a & new \\
\hline HD 289114 & 8.98 & $\mathrm{~K} 2 \mathrm{~V}$ & HD 4628 & $\mathrm{~K} 2 \mathrm{~V}$ & 0.8 & & & known \\
\hline NGC 2264 VAS 92 & 12.38 & G5V & HD 190360 & G6IV & 0.9 & $\log \frac{L_{X}}{L_{\mathrm{b} \mid}}=-3.89$ & $\mathrm{~b}$ & new \\
\hline HD 51478 & 8.08 & M3III & HD 2411 & M3III & 3.0 & & & known \\
\hline 1E0650.4-0520 & 12.60 & $\mathrm{M} 2 \mathrm{~V}$ & HD 95735 & M2V & 3.1 & $\log F_{X}=-13.57\left(\mathrm{Wm}^{-2}\right)$ & $\mathrm{b}$ & new \\
\hline HD 52690 & 6.52 & M3V & HD 95735 & M2V & 3.3 & & & new \\
\hline 1E0627.3-0245 & 13.80 & M4III & HD 130144 & M5III & 6.6 & $\log F_{X}=-13.91\left(W^{-2}\right)$ & $\mathrm{b}$ & new \\
\hline IP Mon & 13.44 & $\mathrm{~K} 2 \mathrm{~V}$ & $61 \mathrm{CygA}$ & $\mathrm{K} 5 \mathrm{~V}$ & 6.7 & $\log \frac{L_{X}}{L_{\mathrm{bol}}}=-3.23$ & $\mathrm{~b}$ & known \\
\hline IO Mon & 13.77 & $\mathrm{~K} 2 \mathrm{~V}$ & $61 \mathrm{CygA}$ & K5V & 18.2 & $\log \frac{L_{X}}{L_{\mathrm{h} \mid}}=-3.31$ & $\mathrm{~b}$ & known \\
\hline MM Mon & 14.14 & K7V & $61 \mathrm{CygA}$ & K5V & 29.8 & $\log \frac{L_{X}}{L_{\mathrm{b} \mid}}=-2.79$ & $\mathrm{~b}$ & known \\
\hline MO Mon & 13.65 & K7V & $61 \mathrm{CygA}$ & $\mathrm{K} 5 \mathrm{~V}$ & 42.7 & $\log \frac{L_{\mathrm{X}}}{L_{\mathrm{bol}}}=-3.10$ & $\mathrm{~b}$ & known \\
\hline
\end{tabular}

a: From ROSAT-PSPC ; b: from XMM-Newton EPIC.

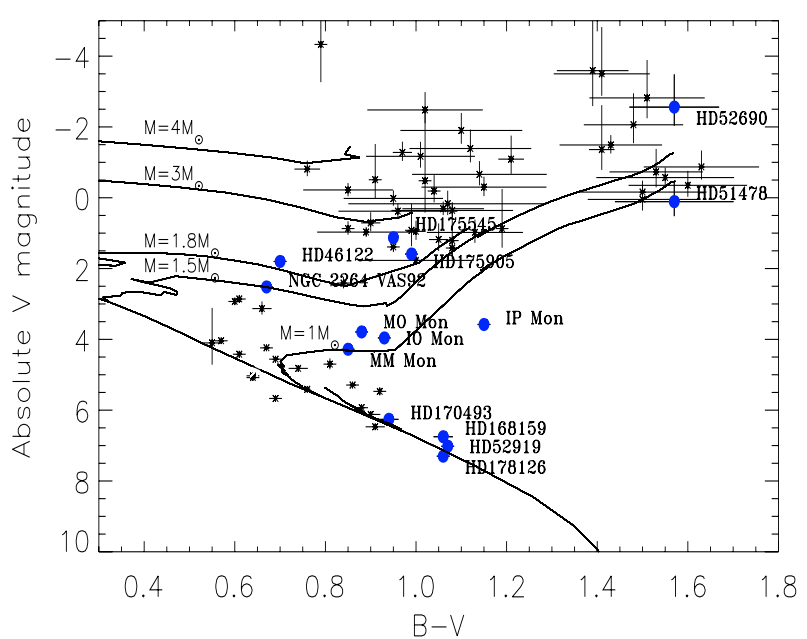

Fig. 3. HR diagram of program stars (asterisks) with known parallax. Bullets indicate the $\mathrm{H} \alpha$ emission stars. The ZAMS and a sample of evolutionary tracks for $Z=0.019$ from Girardi et al. (2000) are also plotted.

agreement with the spectral class assigned by one or both mentioned methods. Only in the case of HD 52690 did the M1.5/3V spectral class assigned by both cross-correlation and ANN methods turn out to be inconsistent with its absolute magnitude, which is typical of a giant star. For 5 targets, the color excess derived from $J H K$ magnitudes was inconsistent with the assigned spectral type. In these cases a $B-V$ color consistent with the spectral type was found by assuming $E(B-V)=0$. In Table 5 we list the absolute magnitude $M_{\mathrm{V}}$ and its uncertainty, intrinsic color $(B-V)_{0}$, color excess $E(B-V)$ as derived from the $E(J-H)$ and $E(H-K)$ color excess according to Cardelli et al. (1989) and its uncertainty and the finally assigned MK class. Additional information on metallicity and evolutionary stage was retrieved by using evolutionary tracks with different metallicities and looking for the one best-fitting either the absolute magnitude or $B-V$ color. For instance, we notice that this approach shows that the cross-correlation method assigns the correct luminosity class more accurately than ANN method. For the remaining 107 targets with no parallax or inaccurate parallax and/or $J H K$ magnitudes, we assigned the MK spectral class with the highest correlation coefficient according to Table 3 . The MK spectral classes we assigned generally match the MK classes given by the SIMBAD database, most of which come from bibliographical surveys, e.g., by Jaschek et al. (1964), as well as from the Michigan Catalogues of Two-Dimensional Spectral Types for the HD stars (Houk \& Cowley 1975). There are a few program stars, however, for which we found very different luminosity classes or spectral types (see Cols. 2 and 4 of Table 5). The complete series of the observed stellar spectra, each along with the finally assigned best-fitting template spectrum, are plotted in Figs. 7-12, which are only available in electronic format.

\subsection{The active stars}

\subsubsection{The excess $\mathrm{H} \alpha$ from spectral subtraction}

The spectral subtraction technique (SST) is a method of estimating the excess $\mathrm{H} \alpha$ emission of a chromospherically active star. The spectrum of the inactive reference star of the same spectral type and luminosity class as the program star is matched and then later subtracted. The residual spectrum obtained by subtracting inactive spectrum is expected to contain only the active chromosphere contribution to the $\mathrm{H} \alpha$ line (Montes et al. 1995, 1997; Barden 1985; Huenemoerde et al. 1989). In practice, it is difficult to get truly chromospherically inactive stars, because all late-type stars are active to some extent. However, from Montes et al. (1995, 1997) and Montes et al. (1999), we selected a list of inactive stars of different spectral classes and observed them with the same instrumental setup as was used 
for the program stars. The spectral subtraction was performed as follows:

1. Both the program and the inactive star's spectra were normalized with respect to the continuum flux around $\mathrm{H} \alpha$.

2. Wavelength and flux shifts were applied whenever needed to match both observed and inactive star spectra.

3. Whenever xy shifts were not enough to match both spectra and a gradient in the program or the inactive star's continuum spectrum was revealed, then it was removed by tilting via a linear regression fit.

4. The difference between template and program star spectra was computed and the strength of excess $\mathrm{H} \alpha$ emission was measured from residual plots.

Our SST analysis reveals that 20 of 180 stars have weak to strong $\mathrm{H} \alpha$ emission in the residual spectrum (see Fig. 6). These result to be about $7 \%$ of the stars in the observed BS sample and about $37 \%$ of the stars in the observed WS sample. The latter result is due to the selection of the target stars in the WS sample that was strongly biased toward already known active stars. Only 2 G-type stars are found to have $\mathrm{H} \alpha$ emission among our program stars. Among the 20 stars showing $\mathrm{H} \alpha$ excess, 13 we detected as chromospherically active for the first time. These stars deserve further follow-up high-resolution observations to better characterize either physical parameters or magnetic activity.

\subsubsection{X-ray activity}

To better characterize the magnetic activity behavior of our targets, we also computed the X-ray to bolometric luminosity ratio, which indicates the presence and level of magnetic activity at the coronal level.

Out of 180 target stars, only 40 stars were detected as X-ray sources during the ROSAT All Sky Survey (RASS). Count rates $\mathrm{H}$ and $\mathrm{S}$ in the hard $(0.4-2.0 \mathrm{KeV})$ and soft $(0.1-0.4 \mathrm{KeV})$ ROSAT-PSPC pulse height channels, respectively, the hardness ratio (HR), and associated errors have been retrieved from the ROSAT All-Sky Survey Faint Source Catalogue (Voges et al. 1999). In order to calculate the X-ray luminosity of these stars, we estimated a count rate-to-energy flux conversion factor (CF) as a function of the hardness ratio following the linear regression curve published by Schmitt et al. (1995) of CF as a function of HR. The uncertainty on the X-ray luminosity was estimated by taking into account the errors on the stellar parallax, count rate, hardness ratio, and intrinsic stellar color.

The X-ray luminosities of the WS sample in the field of the NGC 2264 open cluster are taken from the XMM-Newton EPIC survey (Dahm \& Simon 2005). The bolometric correction to compute the bolometric luminosity $L_{\mathrm{bol}}$ was retrieved from Schmidt-Kaler (1982). The uncertainty on the ratio of the X-ray to bolometric luminosities was assumed to be the same as calculated for the X-ray luminosity, the error on bolometric luminosity being negligible compared to the error on X-ray luminosity. The derived $L_{\mathrm{X}} / L_{\mathrm{bol}}$ values are listed in Col. 7 of Table 5 .

It is known that X-ray and $\mathrm{H} \alpha$ emissions are manifestations of magnetic activity at two different levels of the stellar atmosphere, corona and chromosphere and that they have their common origin in a hydromagnetic dynamo operating just beneath the external convection zone (e.g. Schüssler 1983). As expected from theoretical considerations, both X-ray and $\mathrm{H} \alpha$ emission levels are related to the dynamo efficiency and, therefore, are expected to be correlated to each other, as already observed for activity indicators at different atmospheric levels (e.g. Messina et al. 2003). For a small subsample of targets we have

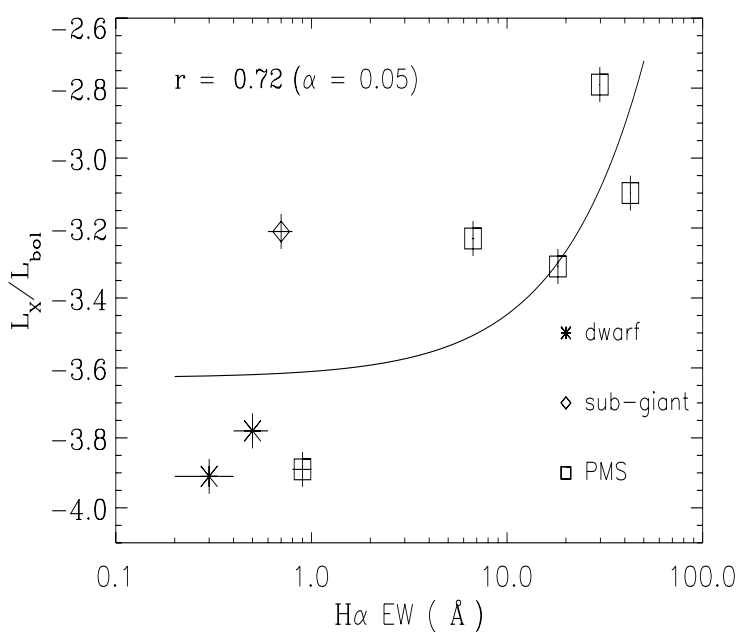

Fig. 4. $L_{\mathrm{X}} / L_{\text {bol }}$ vs. $\mathrm{H} \alpha$ EW for $\mathrm{H} \alpha$ emission stars. The solid line is a linear fit to the data. The linear correlation coefficient ( $r$ ) and its significance level $(\alpha)$ are given in the label. A logarithmic scale is used for the $X$-axis.

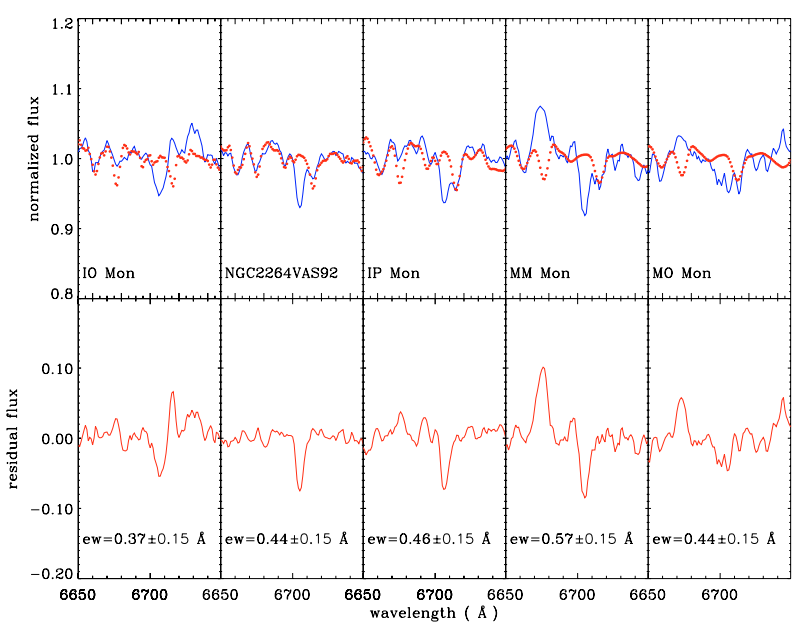

Fig. 5. Top panels: normalized spectra of program stars with evidence of the Li $6708 \AA$ line (continuous line) and non-active template spectra (small filled circles) used for SST. Bottom panels: the residual flux from the spectral subtraction technique.

the measured $\mathrm{H} \alpha \mathrm{EW}$, as well as the computed ratio of X-ray to bolometric luminosities. The ROSAT X-ray luminosities plotted in Fig. 4 were corrected in order to take into account the difference in the energy bands measured by ROSAT with respect to XMM-Newton. We find that these quantities have a linear correlation coefficient $r=0.72$ with a significance level: $\alpha=0.05$. With the exception of the subgiant HD 46122, which significantly deviates from the general trend showed by the other stars, in Fig. 4, IP Mon, IO Mon, MM Mon, MO Mon, and NGC 2264 Vas 92, all of which are in the field of the NGC2264 cluster, show a level of activity/emission that is generally higher than MS field stars.

\subsection{Members of the NGC 2264 open cluster}

IP Mon, IO Mon, MM Mon, MO Mon, NGC 2264 Vas 10 are situated within the very young cluster NGC 2264 ( $~ 9$ Myr). Our spectral analysis shows that all these stars have the $\mathrm{H} \alpha$ excess and, with the exclusion of NGC 2264 Vas 10, evidence of the Li I blend at $6707.810 \AA$. To remove the contribution from the 

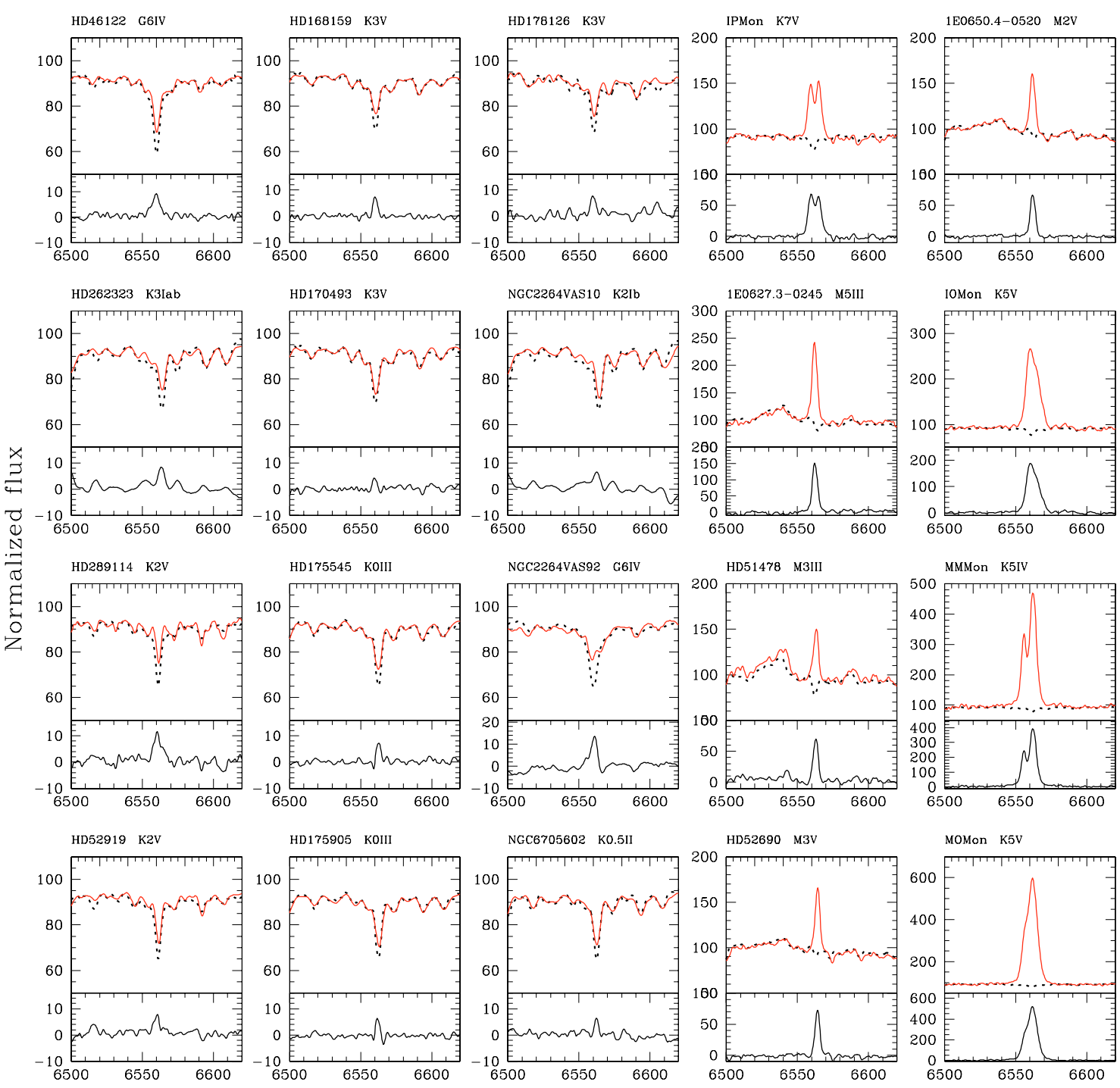

Wavelength (in $\AA$ )

Fig. 6. Results of the spectral subtraction.

Fe I line at $6707.441 \AA$ and to properly measure the Li line EW, we used the SST, following the same procedure as in the case of the $\mathrm{H} \alpha$ line in Sect. 3.2.1. In the top panels of Fig. 5, the spectra of the mentioned stars are plotted along with their template spectra. As shown in the bottom panels, the residual flux of all these stars shows clear evidence of the Li line. The contemporary presence of $\mathrm{H} \alpha$ emission, of Li I $6707.810 \AA$ absorption, and of X-ray emission are all characteristics of the PMS stage, hence our findings give strong support to their membership to NGC 2264 open cluster. Although NGC 2264 Vas 92 has the lowest $\mathrm{H} \alpha$ emission level among these 5 stars, it can be confidently considered as a cluster member. In fact, there are examples of stars that are confirmed PMS WTT stars and whose $\mathrm{H} \alpha$ line is even in absorption (see, e.g. Spezzi et al. 2007, and references therein). To place these targets on the HR diagram in Fig. 3 and make a comparison with evolutionary tracks, we computed absolute magnitudes and dereddened colors assuming a distance of $760 \mathrm{pc}$ and a $E(B-V)=0.15$ color excess according to Rebull et al. (2002). For instance, IO Mon, MM Mon, and MO Mon are the strongest $\mathrm{H} \alpha$ emission stars in our sample with an emission level up to 3,4 , and 5 times, respectively, stronger than the local continuum. From a comparison with the
H $\alpha$ EWs derived by Dahm \& Simon (2005) for IP Mon, IO Mon, MM Mon, and MO Mon, it is possible to argue that the level of $\mathrm{H} \alpha$ emission is highly variable for these PMS stars, with variations up to $\sim 30 \%$ for MO Mon, the strongest $\mathrm{H} \alpha$ emission source in our sample. The MM Mon residual flux from SST is double-peaked; the reason for this behavior deserves further investigation by means of high-resolution spectroscopy.

\section{Conclusions}

In this paper we have described our exploration of the chromospheric activity in a sample of late-type stars. The important findings of the spectral subtraction technique used to characterize the $\mathrm{H} \alpha$ activity and the MK spectral classification of 180 stars in the COROT fields of view are as follows:

1. The MK classification carried out independently by us using our medium-resolution red spectra confirm the MK classification given in SIMBAD for most program stars.

2. Twenty out of 180 observed stars show the presence of weak to strong $\mathrm{H} \alpha$ emission. Thirteen stars are newly discovered chromospherically active stars. 
3. On the basis of our survey of an unbiased sample of BS, the number of active stars in the COROT fields of view is in the range $4.6 \%-8.6 \%(1 \sigma)$.

Magnetic activity is expected to make the discovery of planetary transits from the COROT photometry very challenging. Therefore, the development of techniques (see e.g. Lanza et al. 2005; Moutou et al. 2005) to model and then to remove the effects of activity-induced variability from light curves is extremely important in view of the high percentage of active stars occurring among late-type dwarfs.

Acknowledgements. The Observations reported in this article were obtained using the 2-m Himalayan Chandra Telescope of the Indian Astronomical Observatory, Hanle, the high-altitude station of the Indian Institute of Astrophysics, Bangalore, India. We thank the staff at the IAO and Ramya R. and Jessi J. for their active support during the course of the observations. This work was also supported by the Italian Ministero degli Affari Esteri (MAE) and dell'Università e Ricerca (MUR) and by the Indian Ministery of Science and Technology. The extensive use of the SIMBAD and ADS databases operated by the CDS center, Strasbourg, France, is gratefully acknowledged. This publication makes use of data products from the Two Micron All Sky Survey, which is a joint project of the University of Massachusetts and the Infrared Processing and Analysis Center/California Institute of Technology, funded by the National Aeronautics and Space Administration and the National Science Foundation.

\section{References}

Aigrain, S., Favata, F., \& Gilmore J. 2004, A\&A, 414, 1139

Baglin, A., Vauclair, G., \& The COROT Team 2000, JAp\&A, 21, 19

Bailer-Jones, C. A. L. 1997, PASP, 109, 932

Bailer-Jones, C. A. L., Irwin, M., \& von Hippel, T. 1998, MNRAS, 298, 361

Barden, S. C. 1985, ApJ, 295, 162

Bessel, M. S., \& Brett, J. M. 1988, PASP, 100, 1134

Cardelli, J. A., Clayton, G. C., \& Mathis, J. S. 1989, ApJ, 345, 245

Chisholm, J. R., Harnden, F. R., Jr., Schachter, J. F., et al. 1999, AJ, 117

Cutri, R. M., Skrutskie, M. F., Van Dyk, S., et al. 2003, 2MASS All Sky Catalog of point sources, University of Massachusetts and Infrared Processing and Analysis Center (IPAC/California Institute of Technology)

Dahm, S. E., \& Simon, T. 2005, AJ, 129, 829

Danks, A., \& Dennefeld, M. 1994, PASP, 106, 382
Frasca, A., Alcalá, J. M., Covino, E., et al. 2003, A\&A, 405, 149

Girardi, L., Bressan, A., Bertelli, G., \& Chiosi, C. 2000, A\&AS, 141, 371

Gilmore, G. 2001, Proc, 2nd Eddington workshop, Stellar structure and habitable planet finding, ed. F. Favata, \& D. Galadi-Enriquez

Gulati, R. K., Gupta, R., Gothoskar, P., et al. 1994, ApJ, 426, 340

Jaschek, C., Conde, H., \& de Sierra, A. C. 1964, Publ. La Plata Obs. Ser. Astron., 28,2

Hog, E., Fabricius, C., Makarov, V. V., et al. 2000, A\&A, 355, 27

Houk, N., \& Cowley, A. P. 1975, Michigan Catalogue of two-dimensional spectral types for the HD star (Ann Arbor: University of Michigan, Department of Astronomy)

Huenemoerder, D. P., Ramsey, L. W., \& Buzasi, D. L. 1989, AJ, 98, 2264

Lanza, A. F., Rodonò, M., \& Pagano, I. 2004, A\&A, 425, 707

Lanza, A. F., Rodonò, M., Pagano, I., Barge, P., \& Llebaria, A. 2003, A\&A, 403, 1135

Lanza, A. F., Messina, S., Rodonò, M., \& Pagano, I. 2005, AN, 327, 21

Le Borgne, J.-F., Bruzual, G., Pello, R., et al. 2003, A\&A, 402, 433

Messina, S., Pizzolato, N., Guinan, E. F., \& Rodonò, M. 2003, A\&A, 410, 671

Montes, D., Fernandez-Figueroa , M. J., de Castro, E., et al. 1995, A\&A, 294, 165.

Montes, D., Fernandez-F., M. J., de Castro, E., \& Sanz-F., J. 1997, A\&AS, 125, 263

Montes, D., Ramsey, L. W., \& Welty, A. D. 1999, ApJS, 123, 2

Moutou, C., Pont, F., Barge, P., et al. 2005, A\&A, 437, 355

Pagano, I., et al. in preparation

Perryman, M. A. C., Brown, A. G. A., Lebreton, Y., et al. 1998, A\&A, 331, 81

Poretti, E., Garrido, R., Amado, P. J., et al. 2003, A\&A, 406, 203

Poretti, E., Alonso, R., Amado, P. J., et al. 2005, A\&A,

Ramirez, S. V., Rebull, L., Stauffer, J., et al. 2004, AJ, 127, 2659

Rebull, L. M., Makidon, R. B., Strom, S. E., et al. 2002, AJ, 123, 1528

Singh, H. P., Gulati, R. K., \& Gupta R. 1998, MNRAS, 295, 312

Schmidt-Kaler, Th. 1982, Landolt-Börnstein: numerical data, ed. K. Schaifers, \& H. H. Voigt (Springer-Verlag)

Schüssler, M. 1983, in Solar and stellar magnetic fields, ed. J. O Stenflo (Dordrecht: Reidel), IAU Symp., 102, 213

Schmitt, J. H. M. M., Fleming, T. A., \& Giampapa, M. S. 1995, ApJ, 430, 392

Solano, E., Catala, C., Garrido, R., et al. 2005, AJ, 129, 547

Spezzi, L., Alcalà, J. M., Frasca, A., Covino, E., \& Gandolfi, D. 2007, A\&A, in press

Voges, W., Aschenbach, B., Boller, Th., Braeuninger, H., \& Briel, U. 1999, A\&A, 349, 389

Weiss, W. W., Aerts, C., Aigrain, S., et al. 2003, Proc, 2nd Eddington workshop, Stellar structure and habitable planet finding, ed. F. Favata, \& S. Aigrain 